Supporting Information

For

\title{
Improving the Photovoltaic Performance and Mechanical Stability of Flexible All-Polymer Solar Cells via Tailoring Intermolecular Interactions
}

Minjun Kim, ${ }^{1 \neq}$ Hong Il Kim, ${ }^{1 \neq}$ Seung Un Ryu, ${ }^{1}$ Sung Yun Son, ${ }^{1}$ Sang Ah Park, ${ }^{1}$ Nasir Khan, ${ }^{2}$ Won Suk Shin, ${ }^{2}$ Chang Eun Song, ${ }^{2^{*}}$ and Taiho Park ${ }^{1^{*}}$

${ }^{1}$ Department of Chemical Engineering, Pohang University of Science and Technology, 77 Cheongam-Ro, Nam-Gu, Pohang, Gyeongbuk, 37673, Korea

${ }^{2}$ Energy Materials Research Center, Korea Research Institute of Chemical Technology (KRICT), 141 Gajeong-Ro, Yuseong$\mathrm{Gu}$, Daejeon, 34114, Korea

\section{Contents}

Figure S1. Cyclic voltammograms of (a) PBDB-T, (b) PNDI-TVT and (c) PNDI-FTVT, respectively.

Figure S2. DSC thermograms of (a) PNDI-TVT, (b) PNDI-FTVT, and their blends with PBDB$\mathrm{T}$ content of $55 \mathrm{wt} \%$. Heating rate $=10{ }^{\circ} \mathrm{C} \mathrm{\textrm {min } ^ { - 1 }}$.

Figure S3. GIWAXS (a) in-plane and (b) out-of-plane plot of PNDI-TVT and PNDI-FTVT films, respectively.

Figure S4. GIWAXS azimuthal plot of (a) PNDI-TVT and (b) PNDI-FTVT films, respectively.

Figure S5. GIWAXS (a) in-plane and (b) out-of-plane plot of PBDB-T:PNDI-TVT and PBDBT:PNDI-FTVT films, respectively.

Figure S6. (a) $J-V$ and (b) IPCE characteristic curves for PBDB-T:PNDI-TVT and PBDBT:PNDI-FTVT based rigid all-PSCs, respectively.

Figure S7. $J-V$ characteristics for PBDB-T:PNDI-FTVT based all-PSCs with different additive ratio and thermal annealing, respectively.

Figure S8. $J-V$ characteristics for PBDB-T:PNDI-FTVT based all-PSCs with different weight ratio and thermal annealing, respectively. 
Figure S9. SCLC characteristic curves of PBDB-T:PNDI-TVT and PBDB-T:PNDI-FTVT based hole-only devices.

Figure S10. PL spectrum of pristine PBDB-T film excited at $500 \mathrm{~nm}$.

Figure S11. (a) $V_{\mathrm{OC}}$ and (b) FF factors on the light intensity for PBDB-T:PNDI-TVT and PBDBT:PNDI-FTVT based all-PSCs, respectively.

Figure S12. Normalized (a) PCE, (b) $V_{\mathrm{OC}}$, (c) $J_{\mathrm{SC}}$, and (d) FF for PBDB-T:PNDI-TVT and PBDB-T:PNDI-FTVT based flexible all-PSCs for 30 days, respectively.

Figure S13. $J-V$ characteristic curves for PBDB-T:PNDI-TVT and PBDB-T:PNDI-FTVT based flexible all-PSCs with (a) different bending radius for 100 cycles and (b) different bending cycles at $8 \mathrm{~mm}$ bending radius. (c) Optical images for PBDB-T:PNDI-TVT and PBDB-T:PNDI-FTVT based flexible all-PSCs with different bending radius for 100 cycles.

Table S1. Optical and electrochemical properties of PNDI-TVT and PNDI-FTVT, respectively. Table S2. Crystallographic parameters of PNDI-TVT and PNDI-FTVT films.

Table S3. Photovoltaic performances of flexible all-PSCs.

Table S4. Photovoltaic performances of rigid all-PSCs.

Table S5. Summary of the photovoltaic parameters for PBDB-T:PNDI-FTVT based all-PSCs with different additive ratio and thermal annealing, respectively.

Table S6. Summary of the photovoltaic parameters for PBDB-T:PNDI-FTVT based all-PSCs with different weight ratio and thermal annealing, respectively.

Table S7. Summary of the photovoltaic parameters for PBDB-T:PNDI-TVT and PBDBT:PNDI-FTVT based flexible all-PSCs with different bending radius for 100 cycles, respectively. Table S8. Summary of the photovoltaic parameters for PBDB-T:PNDI-TVT and PBDBT:PNDI-FTVT based flexible all-PSCs with different bending cycles at $8 \mathrm{~mm}$ bending radius. 

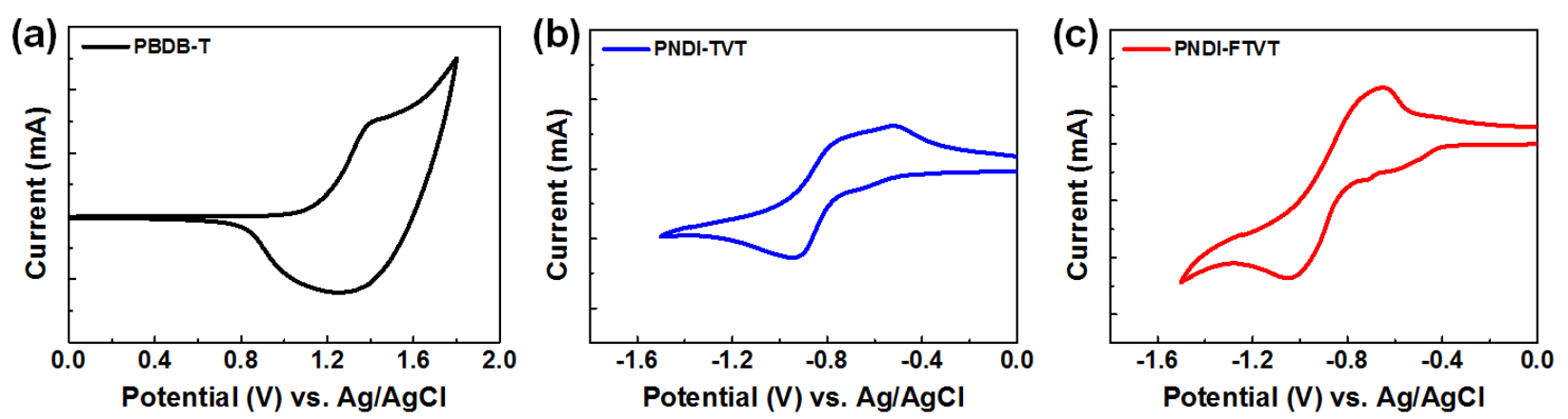

Figure S1. Cyclic voltammograms of (a) PBDB-T, (b) PNDI-TVT and (c) PNDI-FTVT, respectively. 

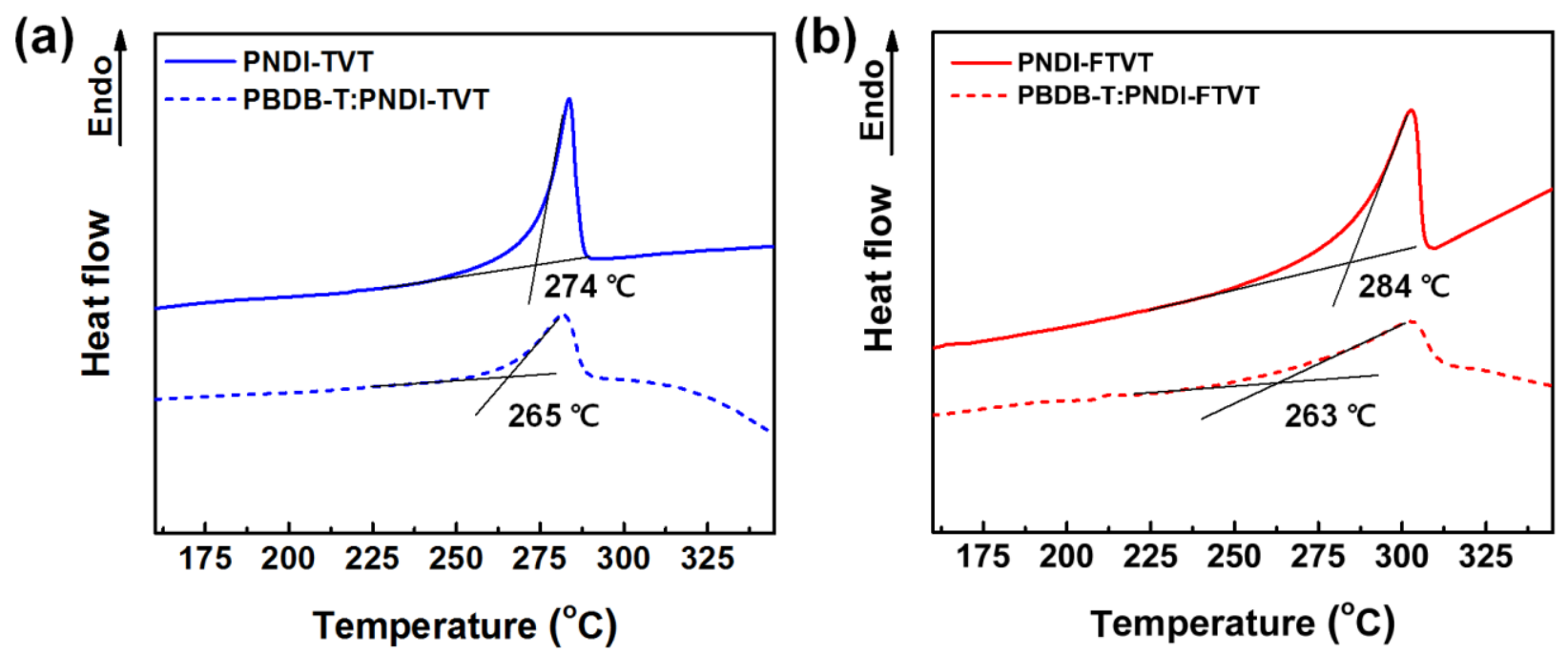

Figure S2. DSC thermograms of (a) PNDI-TVT, (b) PNDI-FTVT, and their blends with PBDB$\mathrm{T}$ content of $55 \mathrm{wt} \%$. Heating rate $=10{ }^{\circ} \mathrm{C} \mathrm{\textrm {min } ^ { - 1 }}$. 

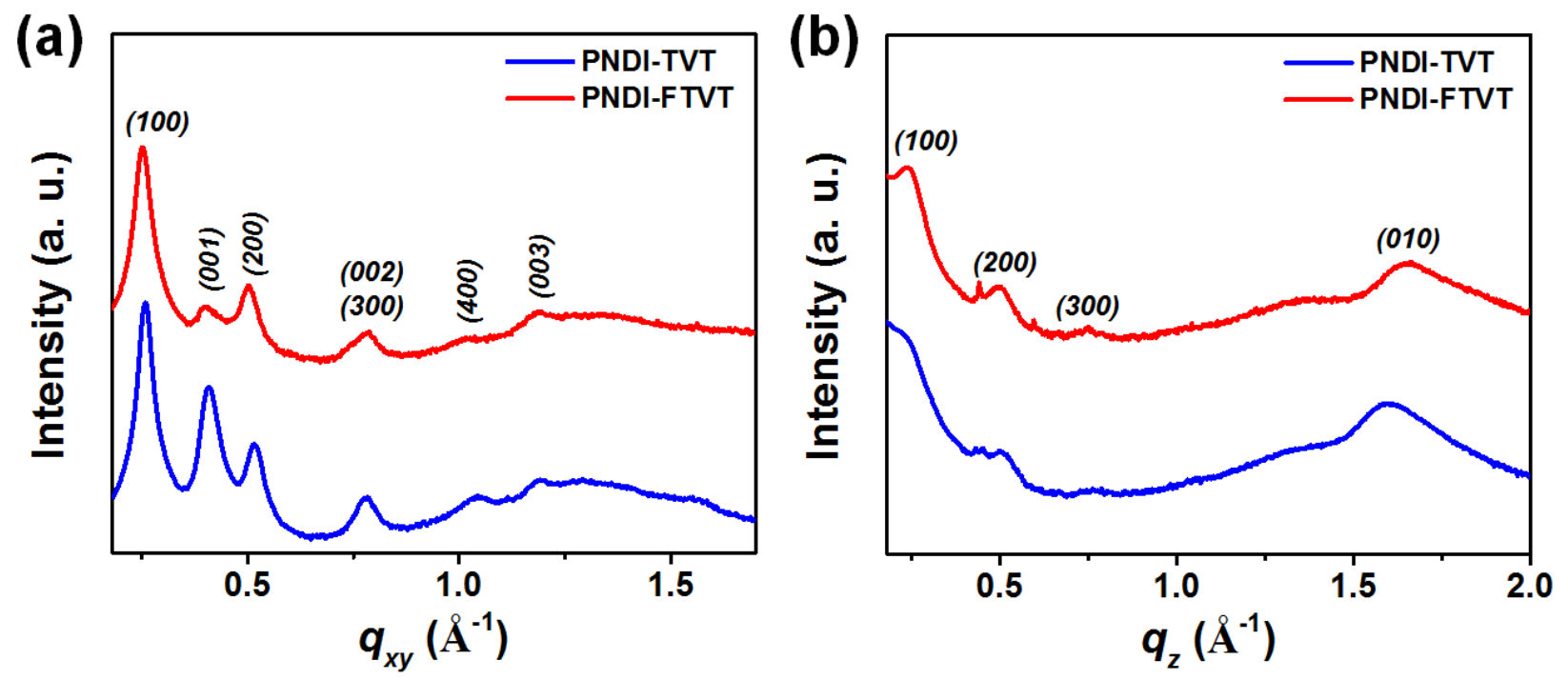

Figure S3. GIWAXS (a) in-plane and (b) out-of-plane plot of PNDI-TVT and PNDI-FTVT films, respectively. 

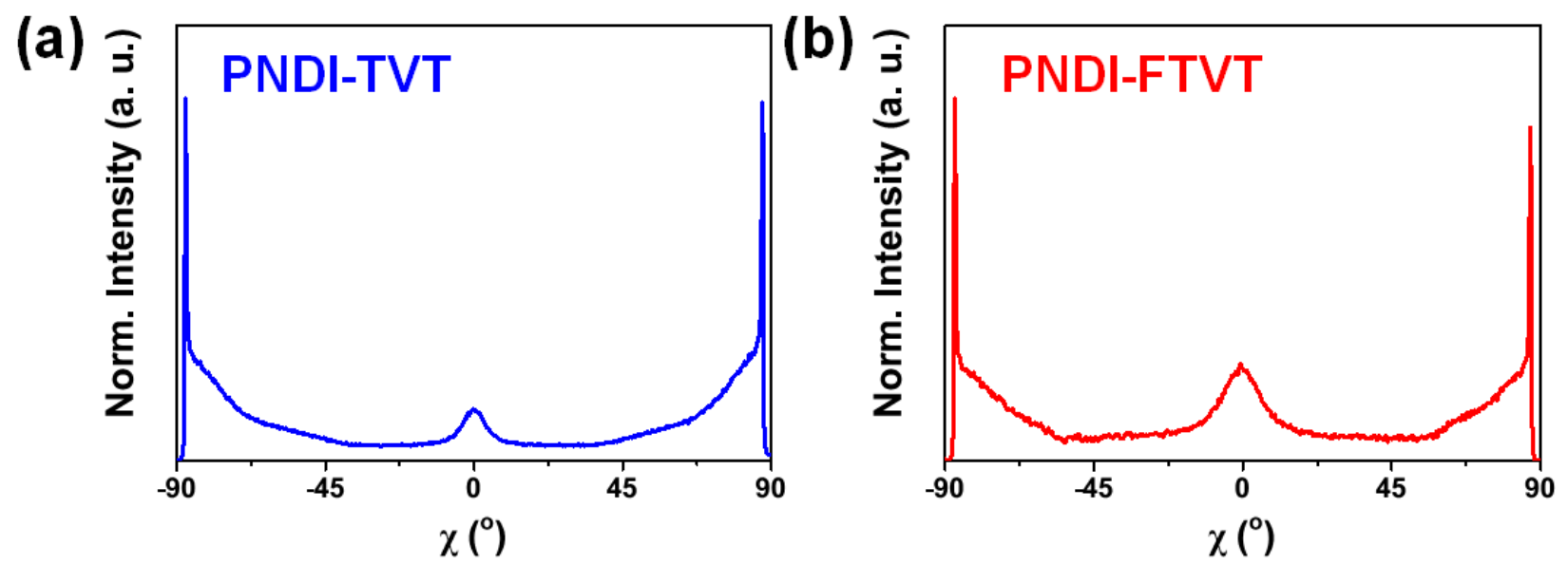

Figure S4. GIWAXS azimuthal plot of (a) PNDI-TVT and (b) PNDI-FTVT films, respectively. 

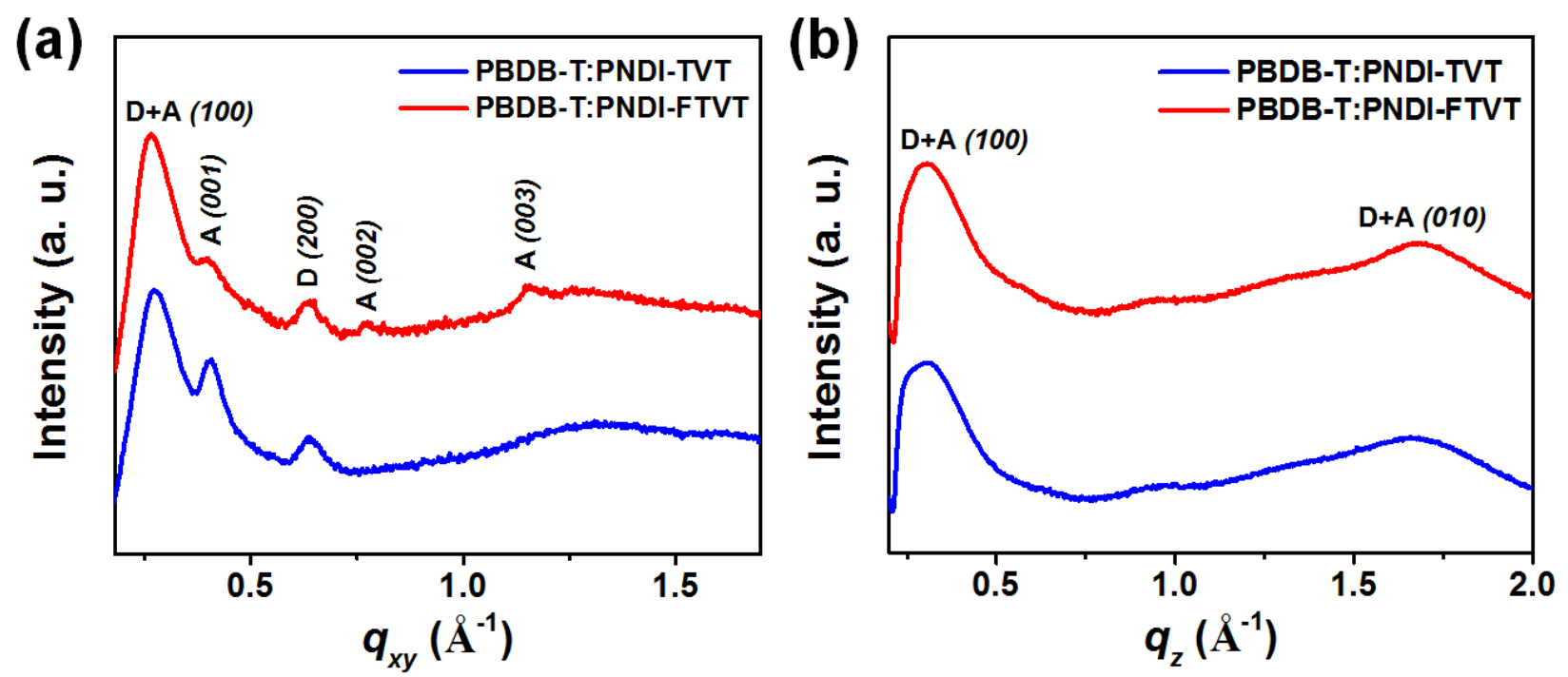

Figure S5. GIWAXS (a) in-plane and (b) out-of-plane plot of PBDB-T:PNDI-TVT and PBDBT:PNDI-FTVT films, respectively. 
(a)

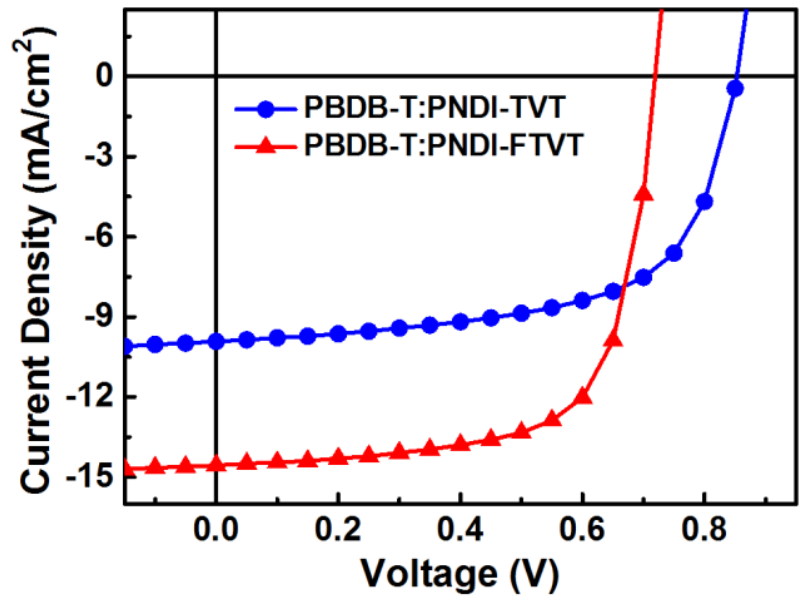

(b)

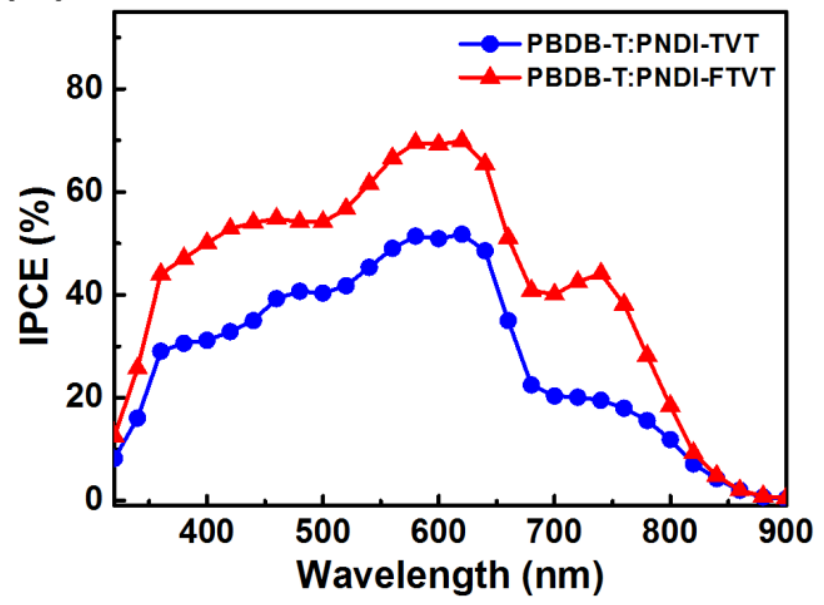

Figure S6. (a) $J-V$ and (b) IPCE characteristic curves for PBDB-T:PNDI-TVT and PBDBT:PNDI-FTVT based rigid all-PSCs, respectively. 


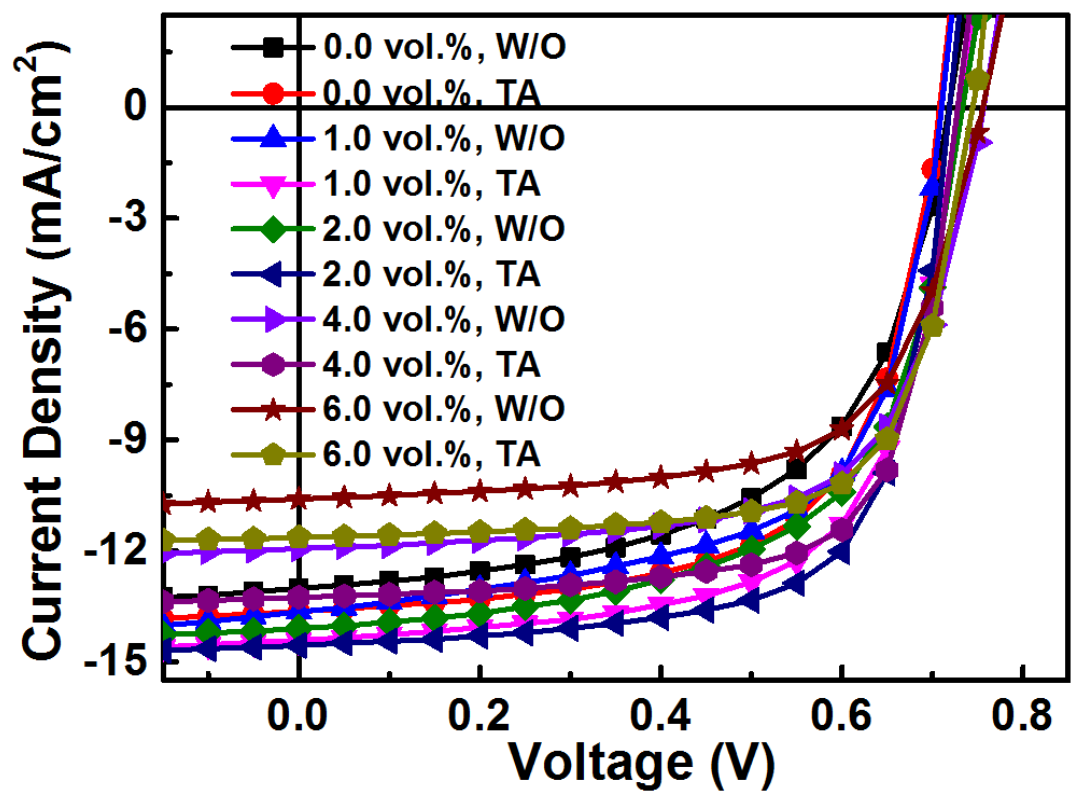

Figure S7. $J-V$ characteristics for PBDB-T:PNDI-FTVT based all-PSCs with different additive ratio and thermal annealing, respectively.

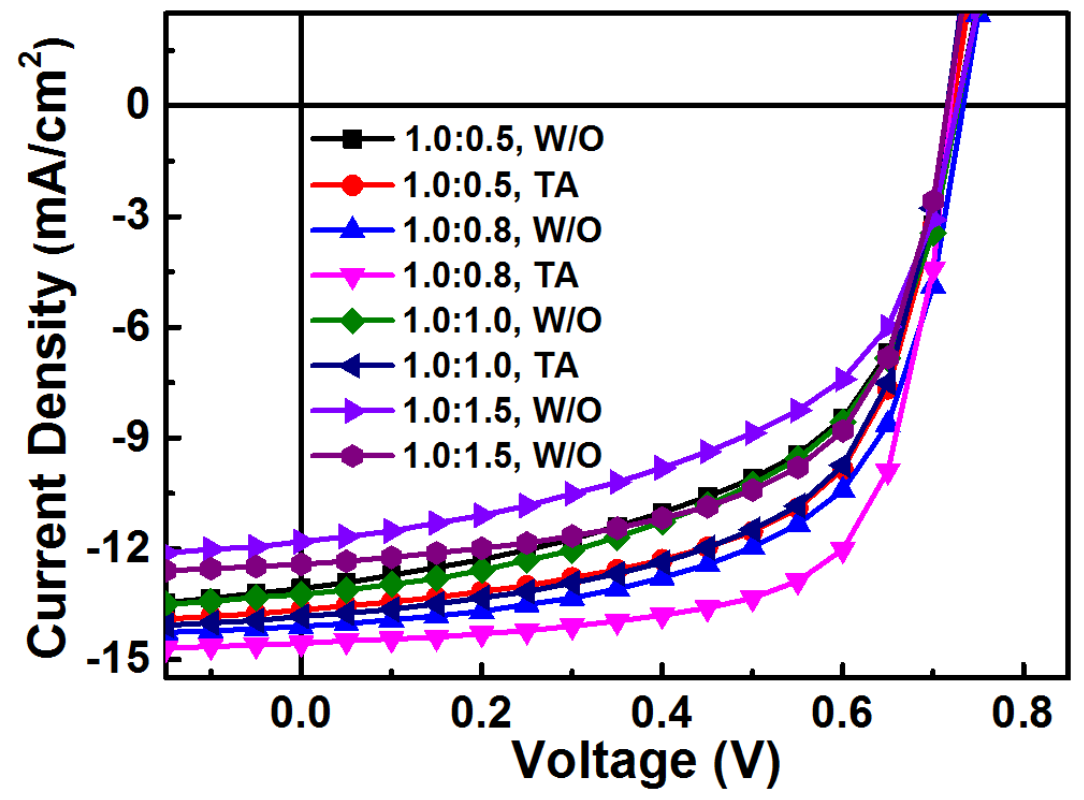

Figure S8. $J-V$ characteristics for PBDB-T:PNDI-FTVT based all-PSCs with different weight ratio and thermal annealing, respectively. 


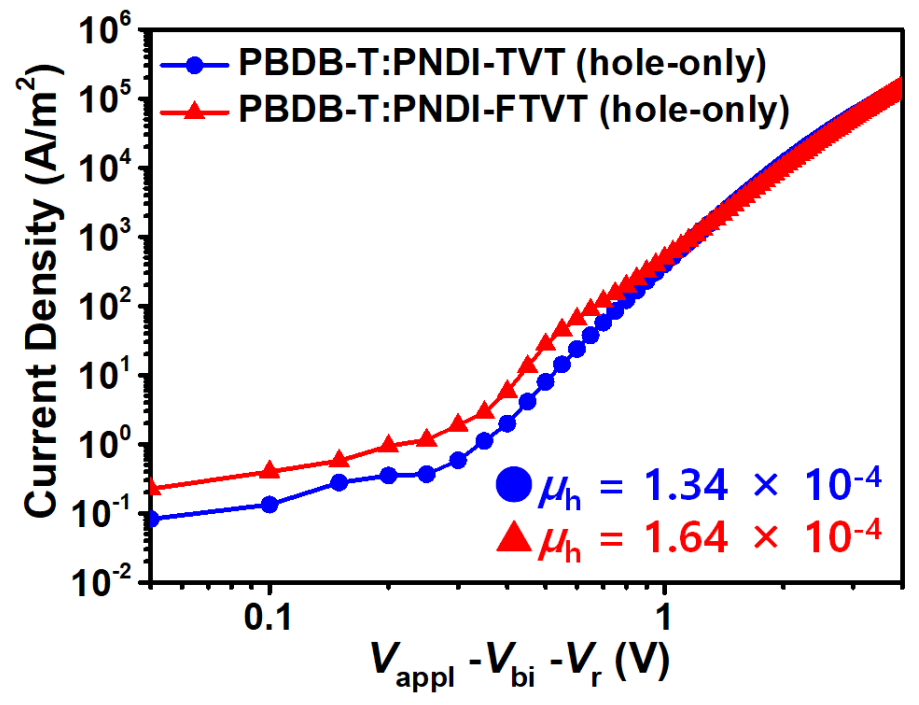

Figure S9. SCLC characteristic curves of PBDB-T:PNDI-TVT and PBDB-T:PNDI-FTVT based hole-only devices. 


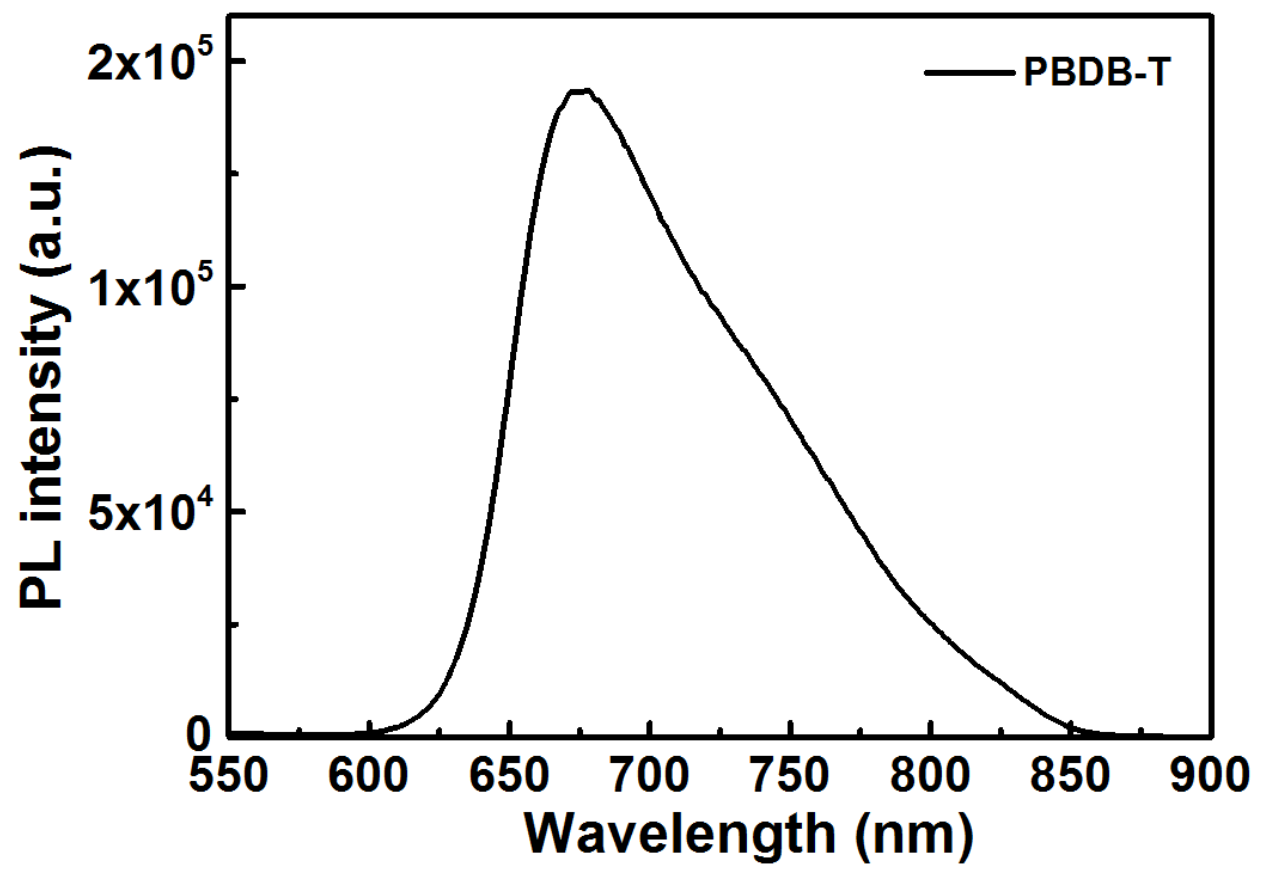

Figure S10. PL spectrum of pristine PBDB-T film excited at $500 \mathrm{~nm}$. 
(a)

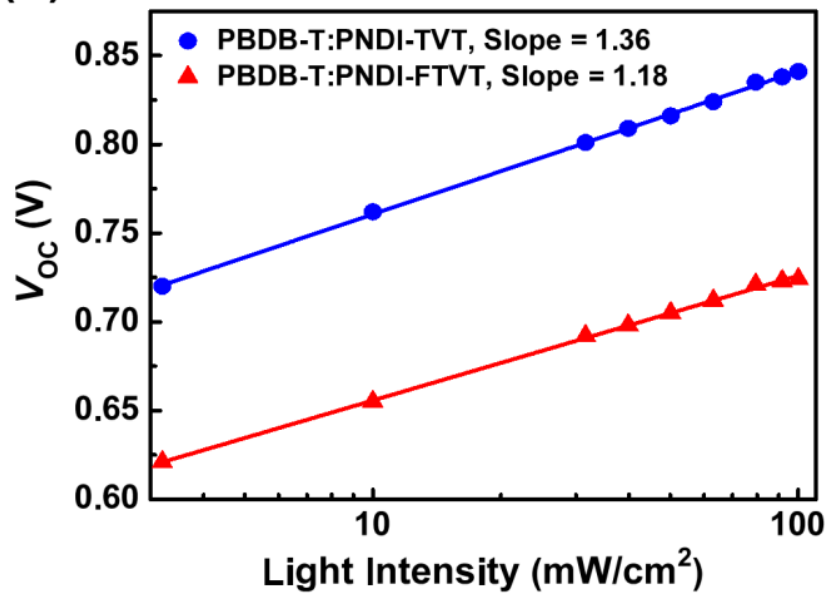

(b)

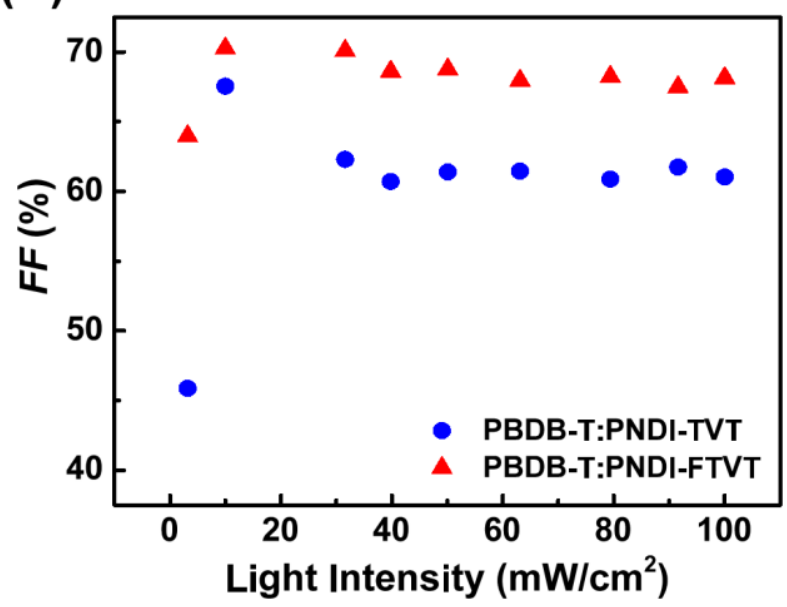

Figure S11. (a) $V_{\mathrm{OC}}$ and (b) $F F$ factors on the light intensity for PBDB-T:PNDI-TVT and PBDB-T:PNDI-FTVT based all-PSCs, respectively. 

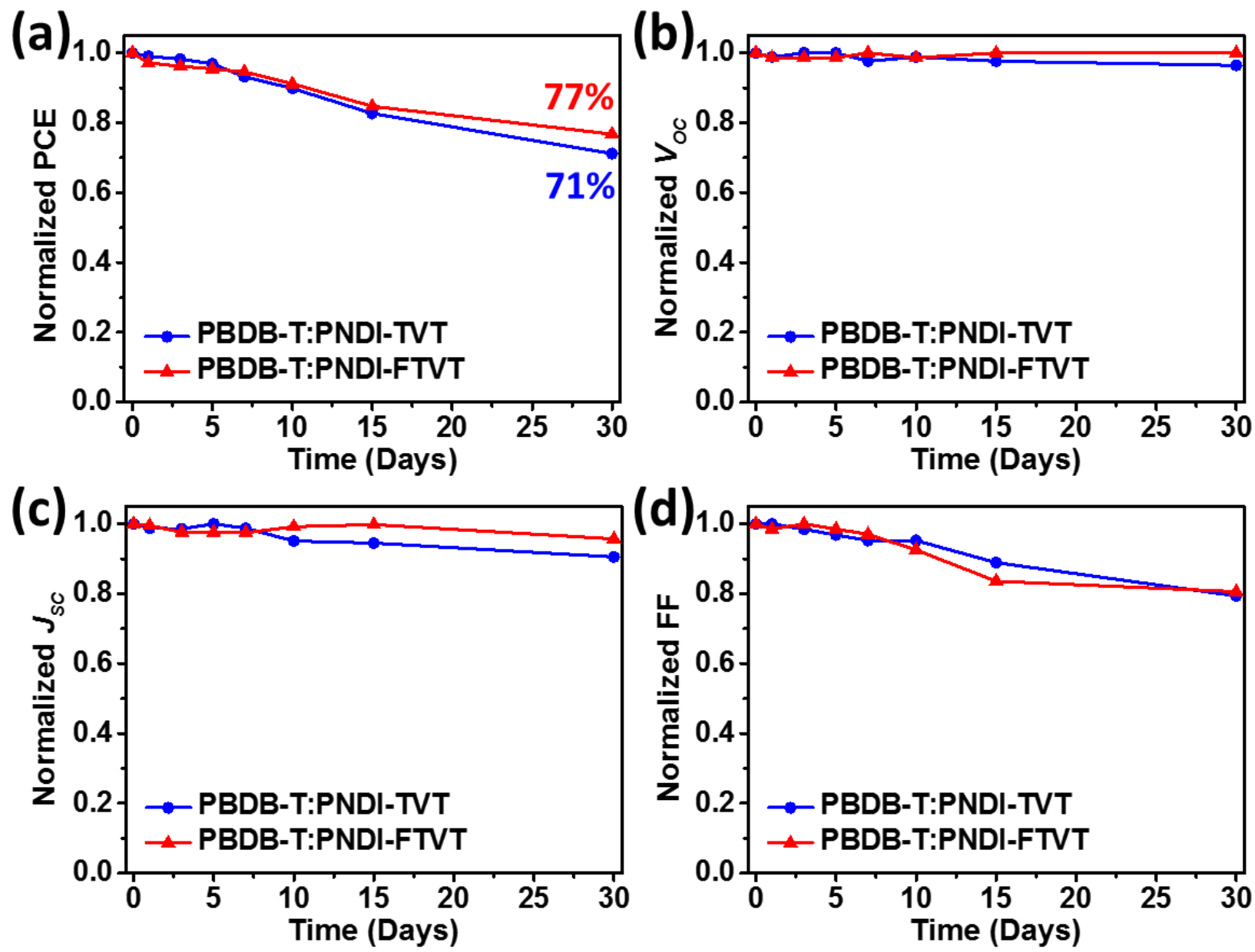

Figure S12. Normalized (a) PCE, (b) $V_{\mathrm{OC}}$, (c) $J_{\mathrm{SC}}$, and (d) FF for PBDB-T:PNDI-TVT and PBDB-T:PNDI-FTVT based flexible all-PSCs for 30 days. 
(a)

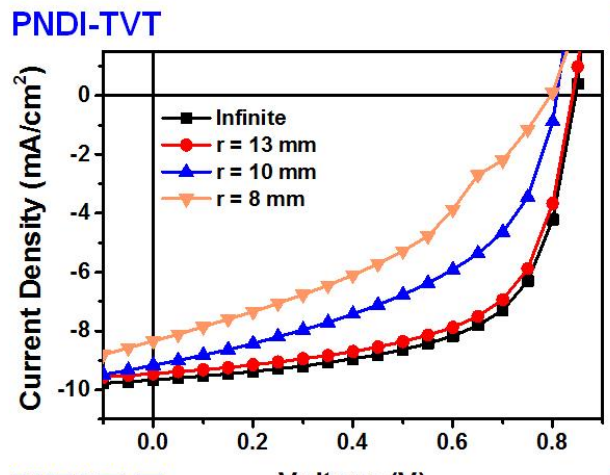

(b)

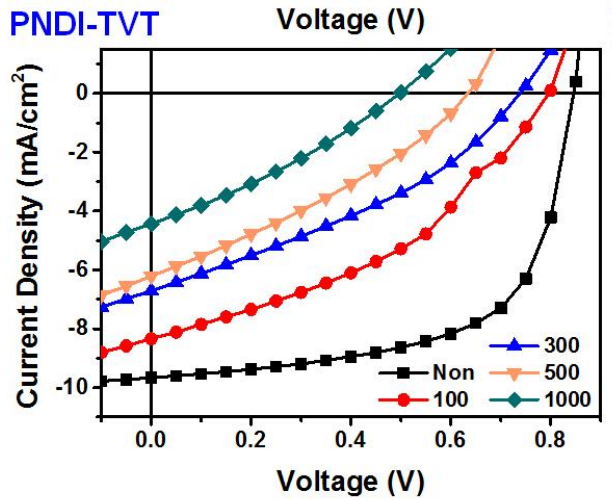

(c)
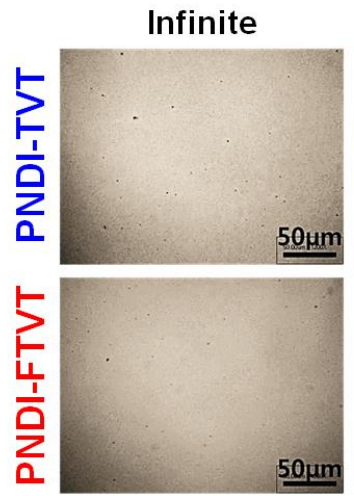

$R=13 \mathrm{~mm}$
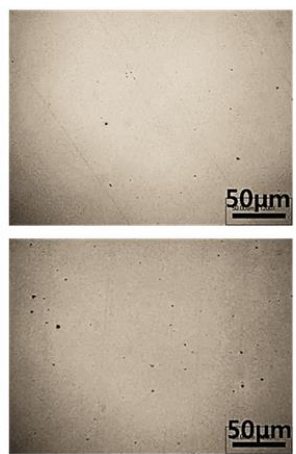

PNDI-FTVT

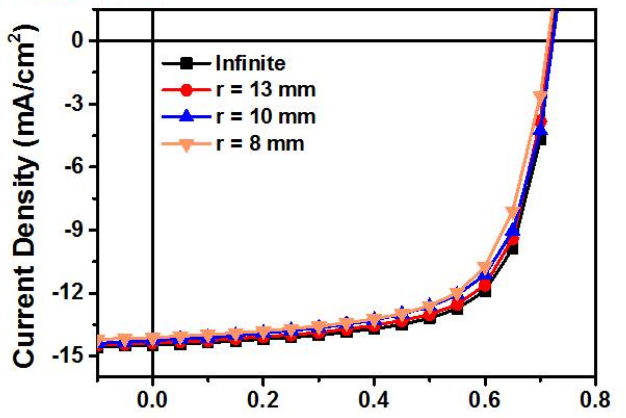

PNDI-FTVT Voltage (V)

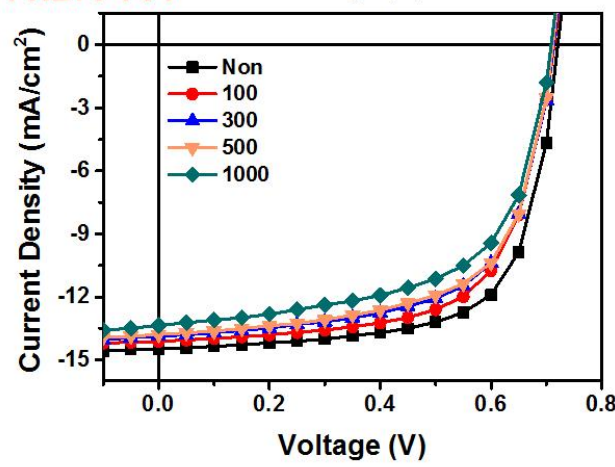

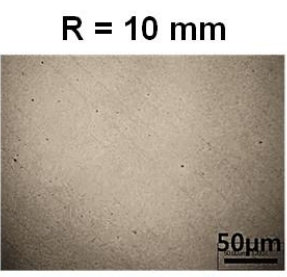

$\mathrm{R}=8 \mathrm{~mm}$
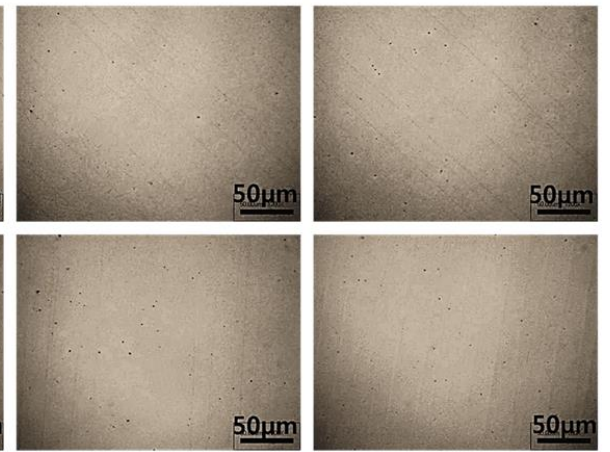

Figure S13. $J-V$ characteristic curves for PBDB-T:PNDI-TVT and PBDB-T:PNDI-FTVT based flexible all-PSCs with (a) different bending radius for 100 cycles and (b) different bending cycles at $8 \mathrm{~mm}$ bending radius. (c) Optical images for PBDB-T:PNDI-TVT and PBDB-T:PNDI-FTVT based flexible all-PSCs with different bending radius for 100 cycles. 
Table S1. Optical and electrochemical properties of PNDI-TVT and PNDI-FTVT, respectively.

\begin{tabular}{ccccc}
\hline $\begin{array}{c}\text { Polymer } \\
\text { Acceptors }\end{array}$ & $\begin{array}{c}\text { LUMO } \\
{[\mathrm{eV}]^{\mathrm{a}}}\end{array}$ & $\begin{array}{c}\text { HOMO } \\
{\left[\mathrm{eV}^{\mathrm{b}}\right.}\end{array}$ & $\begin{array}{c}E_{\mathrm{g}}^{\text {opt }} \\
{\left[\mathrm{eV}^{\mathrm{c}}\right.}\end{array}$ & $\begin{array}{c}\varepsilon \\
{\left[\mathrm{M}^{-1} \mathrm{~cm}^{-1}\right]^{\mathrm{d})}}\end{array}$ \\
\hline PNDI-TVT & -3.89 & -5.36 & 1.47 & $3.88 \times 10^{4}$ \\
PNDI-FTVT & -3.96 & -5.50 & 1.54 & $4.75 \times 10^{4}$ \\
\hline
\end{tabular}

${ }^{\text {a) }}$ LUMO was measured from the $E_{\text {onset }}$ of the first reduction potential relative to ferrocene (4.8 $\mathrm{eV}) ;{ }^{\mathrm{b}} \mathrm{HOMO}=\mathrm{LUMO}-E_{\mathrm{g}}{ }^{\text {opt. }}{ }^{\mathrm{c})} E_{\mathrm{g}}{ }^{\text {opt }}$ was estimated from band edges in the film state; ${ }^{\mathrm{d})}$ Maximum absorption coefficients at $745 \mathrm{~nm}$.

Table S2. Crystallographic parameters of PNDI-TVT and PNDI-FTVT films.

\begin{tabular}{ccccccccc}
\hline \multirow{2}{*}{\begin{tabular}{c} 
Polymer \\
\cline { 2 - 8 } Acceptors
\end{tabular}} & $d_{(100)}$ & $d_{(001)}$ & $L_{\mathrm{T}}^{(h 00)}$ & $L_{\mathrm{T}}^{(00 l)}$ & $d_{(100)}$ & $d_{(010)}$ & $L_{\mathrm{T}}^{((h 00)}$ & $L_{\mathrm{C}}{ }^{(010)}$ \\
& {$[\AA]$} & {$[\AA]$} & {$[\AA]$} & {$[\AA]$} & {$[\AA]$} & {$[\AA]$} & {$[\AA]$} & {$[\AA]$} \\
\hline PNDI-TVT & 24.3 & 15.3 & 279 & 174 & 26.8 & 3.91 & 141 & 28.8 \\
PNDI-FTVT & 25.0 & 15.6 & 224 & 144 & 26.1 & 3.77 & 132 & 23.8 \\
\hline
\end{tabular}

${ }^{\text {a) }}$ True paracrystal size was calculated from the reciprocal value of intercept of $\delta b-h^{2}$ plot; ${ }^{\text {b) }}$ The distortion parameter of paracrystallinity was calculated from the slope of $\delta b-h^{2}$ plot; ${ }^{\text {c) Coherence }}$ length estimated from $\pi-\pi$ stacking diffraction $(010)$ peak.

Table S3. Photovoltaic performances of flexible all-PSCs.

\begin{tabular}{cccccc}
\hline $\begin{array}{c}\text { Polymer } \\
\text { Acceptors }\end{array}$ & $\begin{array}{c}J_{\mathrm{SC}} \\
{\left[\mathrm{mA} \mathrm{cm}^{-2}\right]}\end{array}$ & $\begin{array}{c}\text { Cal. } J_{\mathrm{SC}} \\
{\left[\mathrm{mA} \mathrm{cm}^{-2] \mathrm{a})}\right.}\end{array}$ & $\begin{array}{c}V_{\mathrm{OC}} \\
{[\mathrm{V}]}\end{array}$ & $\begin{array}{c}F F \\
{[\%]}\end{array}$ & $\begin{array}{c}\text { PCE } \\
{[\%]}\end{array}$ \\
\hline PNDI-TVT & 9.67 & 9.43 & 0.85 & 62.0 & $5.11(4.90)^{\mathrm{b})}$ \\
PNDI-FTVT & 14.5 & 14.0 & 0.72 & 68.0 & $7.14(6.95)^{\mathrm{b})}$ \\
\hline
\end{tabular}

PEN/ITO/ZnO NPs/PEIE/PBDB-T:PNDI-TVT or PBDB-T:PNDI-FTVT/MoO $3 / \mathrm{Ag} ;{ }^{\text {a) }}$ The integrated $J_{\mathrm{SC}}$ values are calculated from IPCE data; ${ }^{\mathrm{b})}$ The average PCEs are obtained from over 10 devices.

Table S4. Photovoltaic performances of rigid all-PSCs.

\begin{tabular}{cccccc}
\hline $\begin{array}{c}\text { Polymer } \\
\text { Acceptors }\end{array}$ & $\begin{array}{c}J_{\mathrm{SC}} \\
{\left[\mathrm{mA} \mathrm{cm}^{-2}\right]}\end{array}$ & $\begin{array}{c}\text { Cal. } J_{\mathrm{SC}} \\
{\left[\mathrm{mA} \mathrm{cm}^{-2] \mathrm{a})}\right.}\end{array}$ & $\begin{array}{c}V_{\mathrm{OC}} \\
{[\mathrm{V}]}\end{array}$ & $\begin{array}{c}F F \\
{[\%]}\end{array}$ & $\begin{array}{c}\text { PCE } \\
{[\%]}\end{array}$ \\
\hline PNDI-TVT & 9.92 & 9.69 & 0.85 & 62.0 & $5.27(5.06)^{\mathrm{b})}$ \\
PNDI-FTVT & 14.6 & 14.2 & 0.72 & 69.0 & $7.21(7.04)^{\mathrm{b})}$ \\
\hline
\end{tabular}

Glass/ITO/ZnO NPs/PEIE/PBDB-T:PNDI-TVT or PBDB-T:PNDI-FTVT/MoO $3 / \mathrm{Ag}$; ${ }^{\text {a) }} \mathrm{The}$ integrated $J_{\mathrm{SC}}$ values are calculated from IPCE data; ${ }^{\text {b) }}$ The average PCEs are obtained from over 10 devices. 
Table S5. Summary of the photovoltaic parameters for PBDB-T:PNDI-FTVT based all-PSCs with different additive ratio and thermal annealing, respectively.

\begin{tabular}{|c|c|c|c|c|c|c|}
\hline $\begin{array}{l}\text { Polymer } \\
\text { Acceptor }\end{array}$ & $\begin{array}{c}\text { FPE } \\
\text { Additive }\end{array}$ & $\begin{array}{c}\text { Thermal } \\
\text { Annealing }\end{array}$ & $\begin{array}{c}J_{\mathrm{SC}} \\
{\left[\mathrm{mA} \mathrm{cm}^{-2}\right]}\end{array}$ & $\begin{array}{l}V_{\mathrm{OC}} \\
{[\mathrm{V}]}\end{array}$ & $\begin{array}{l}F F \\
{[\%]}\end{array}$ & $\begin{array}{l}\text { PCE } \\
{[\%]}\end{array}$ \\
\hline \multirow{10}{*}{ PNDI-FTVT } & \multirow{2}{*}{0.0 vol. $\%$} & $\mathrm{~W} / \mathrm{O}$ & 13.0 & 0.72 & 58 & 5.39 \\
\hline & & $120^{\circ} \mathrm{C}, 10 \mathrm{~min}$. & 13.6 & 0.71 & 64 & 6.13 \\
\hline & \multirow{2}{*}{1.0 vol. $\%$} & $\mathrm{~W} / \mathrm{O}$ & 13.7 & 0.71 & 62 & 6.00 \\
\hline & & $120^{\circ} \mathrm{C}, 10 \mathrm{~min}$ & 14.4 & 0.73 & 64 & 6.77 \\
\hline & \multirow{2}{*}{2.0 vol. $\%$} & $\mathrm{~W} / \mathrm{O}$ & 14.1 & 0.73 & 60 & 6.25 \\
\hline & & $120^{\circ} \mathrm{C}, 10 \mathrm{~min}$ & 14.6 & 0.72 & 69 & 7.21 \\
\hline & \multirow{2}{*}{4.0 vol. $\%$} & $\mathrm{~W} / \mathrm{O}$ & 11.9 & 0.76 & 66 & 5.96 \\
\hline & & $120^{\circ} \mathrm{C}, 10 \mathrm{~min}$ & 13.3 & 0.73 & 71 & 6.86 \\
\hline & \multirow{2}{*}{6.0 vol. $\%$} & $\mathrm{~W} / \mathrm{O}$ & 10.6 & 0.76 & 65 & 5.23 \\
\hline & & $120^{\circ} \mathrm{C}, 10 \mathrm{~min}$ & 11.6 & 0.74 & 71 & 6.11 \\
\hline
\end{tabular}

Glass/ITO/ZnO NPs/PEIE/PBDB-T:PNDI-FTVT/MoO 3 /Ag.

Table S6. Summary of the photovoltaic parameters for PBDB-T:PNDI-FTVT based all-PSCs with different weight ratio and thermal annealing, respectively.

\begin{tabular}{|c|c|c|c|c|c|c|}
\hline $\begin{array}{l}\text { Polymer } \\
\text { Acceptor }\end{array}$ & $\begin{array}{c}\text { Weight } \\
\text { Ratio (D:A) }\end{array}$ & $\begin{array}{c}\text { Thermal } \\
\text { Annealing }\end{array}$ & $\begin{array}{c}J_{\mathrm{SC}} \\
{\left[\mathrm{mA} \mathrm{cm}^{-2}\right]}\end{array}$ & $\begin{array}{l}V_{\mathrm{OC}} \\
{[\mathrm{V}]}\end{array}$ & $\begin{array}{l}F F \\
{[\%]}\end{array}$ & $\begin{array}{l}\text { PCE } \\
{[\%]}\end{array}$ \\
\hline \multirow{8}{*}{ PNDI-FTVT } & \multirow{2}{*}{$1.0: 0.5$} & $\mathrm{~W} / \mathrm{O}$ & 13.1 & 0.73 & 55 & 5.19 \\
\hline & & $120^{\circ} \mathrm{C}, 10 \mathrm{~min}$. & 13.7 & 0.72 & 61 & 6.00 \\
\hline & \multirow{2}{*}{$1.0: 0.8$} & $\mathrm{~W} / \mathrm{O}$ & 14.1 & 0.73 & 60 & 6.25 \\
\hline & & $120^{\circ} \mathrm{C}, 10 \mathrm{~min}$ & 14.6 & 0.72 & 69 & 7.21 \\
\hline & \multirow{2}{*}{ 1.0:1.0 } & $\mathrm{W} / \mathrm{O}$ & 13.2 & 0.73 & 55 & 5.26 \\
\hline & & $120^{\circ} \mathrm{C}, 10 \mathrm{~min}$ & 13.8 & 0.72 & 60 & 5.96 \\
\hline & \multirow{2}{*}{$1.0: 1.5$} & $\mathrm{~W} / \mathrm{O}$ & 11.8 & 0.73 & 53 & 4.54 \\
\hline & & $120^{\circ} \mathrm{C}, 10 \mathrm{~min}$ & 12.4 & 0.72 & 61 & 5.39 \\
\hline
\end{tabular}

Glass/ITO/ZnO NPs/PEIE/PBDB-T:PNDI-FTVT/MoO $3 /$ Ag. 
Table S7. Summary of the photovoltaic parameters for PBDB-T:PNDI-TVT and PBDBT:PNDI-FTVT based flexible all-PSCs with different bending radius for 100 cycles, respectively.

\begin{tabular}{cccccc}
\hline Active Layer & $\begin{array}{c}\text { Bending } \\
\text { Radius }\end{array}$ & $\begin{array}{c}V_{\mathrm{OC}} \\
{[\mathrm{V}]}\end{array}$ & $\begin{array}{c}J_{\mathrm{SC}} \\
{\left[\mathrm{mA} \mathrm{cm}^{-2}\right]}\end{array}$ & $\begin{array}{c}\mathrm{FF} \\
{[\%]}\end{array}$ & $\begin{array}{c}\text { PCE } \\
{[\%]}\end{array}$ \\
\hline \multirow{3}{*}{ PBDB-T:PNDI-TVT } & Infinite & 0.85 & 9.67 & 62 & $5.11(4.90)^{\mathrm{a})}$ \\
\cline { 2 - 6 } & $13 \mathrm{~mm}$ & 0.84 & 9.45 & 61 & $4.88(4.55)^{\mathrm{a})}$ \\
\cline { 2 - 6 } & $10 \mathrm{~mm}$ & 0.81 & 9.16 & 48 & $3.55(3.13)^{\mathrm{a})}$ \\
\hline & $8 \mathrm{~mm}$ & 0.79 & 8.34 & 40 & $2.64(2.20)^{\mathrm{a})}$ \\
\hline \multirow{2}{*}{ PBDB-T:PNDI-FTVT } & Infinite & 0.72 & 14.47 & 68 & $7.14(6.95)^{\mathrm{a})}$ \\
\cline { 2 - 6 } & $13 \mathrm{~mm}$ & 0.72 & 14.35 & 68 & $6.97(6.66)^{\mathrm{a})}$ \\
\cline { 2 - 6 } & $10 \mathrm{~mm}$ & 0.72 & 14.24 & 65 & $6.67(6.26)^{\mathrm{a})}$ \\
\hline & $8 \mathrm{~mm}$ & 0.71 & 14.11 & 65 & $6.59(6.18)^{\mathrm{a})}$ \\
\hline
\end{tabular}

a) The average PCEs are obtained from over 10 devices. PEN/ITO/ZnO NPs/PEIE/polymer donor:polymer acceptor/ $\mathrm{MoO}_{3} / \mathrm{Ag}$.

Table S8. Summary of the photovoltaic parameters for PBDB-T:PNDI-TVT and PBDBT:PNDI-FTVT based flexible all-PSCs with different bending cycles at $8 \mathrm{~mm}$ bending radius.

\begin{tabular}{|c|c|c|c|c|c|}
\hline Active Layer & $\begin{array}{c}\text { Bending } \\
\text { Radius }\end{array}$ & $\begin{array}{l}V_{\mathrm{OC}} \\
{[\mathrm{V}]}\end{array}$ & $\begin{array}{c}J_{\mathrm{SC}} \\
{\left[\mathrm{mA} \mathrm{cm}{ }^{-2}\right]}\end{array}$ & $\begin{array}{l}F F \\
{[\%]}\end{array}$ & $\begin{array}{l}\mathrm{PCE} \\
{[\%]}\end{array}$ \\
\hline \multirow{5}{*}{ PBDB-T:PNDI-TVT } & Non & 0.85 & 9.67 & 62 & $5.11(4.90)^{\mathrm{a})}$ \\
\hline & 100 & 0.79 & 8.34 & 40 & $2.64(2.20)^{\mathrm{a})}$ \\
\hline & 300 & 0.74 & 6.70 & 34 & $1.70(1.28)^{\mathrm{a})}$ \\
\hline & 500 & 0.63 & 6.21 & 32 & $1.24(0.90)^{\mathrm{a})}$ \\
\hline & 1000 & 0.50 & 4.44 & 30 & $0.67(0.36)^{\mathrm{a})}$ \\
\hline \multirow{5}{*}{ PBDB-T:PNDI-FTVT } & Non & 0.72 & 14.47 & 68 & $7.14(6.95)^{\mathrm{a})}$ \\
\hline & 100 & 0.71 & 14.11 & 65 & $6.59(6.18)^{\mathrm{a})}$ \\
\hline & 300 & 0.71 & 13.87 & 64 & $6.31(5.95)^{\mathrm{a})}$ \\
\hline & 500 & 0.71 & 13.78 & 64 & $6.25(5.88)^{\mathrm{a})}$ \\
\hline & 1000 & 0.71 & 13.35 & 61 & $5.78(5.39)^{\mathrm{a})}$ \\
\hline
\end{tabular}

a) The average PCEs are obtained from over 10 independent devices. PEN/ITO/ZnO NPs/PEIE/polymer donor:polymer acceptor/ $\mathrm{MoO}_{3} / \mathrm{Ag}$. 